\title{
A Note on Bayesian Analysis of Error Function Distribution under Different Loss Functions
}

\author{
Navid Feroze ${ }^{1, *}$, Muhammad Aslam² \\ ${ }^{1}$ Department of Mathematics and Statistics, Allama Iqbal Open University, Islamabad, Pakistan \\ ${ }^{2}$ Department of Statistics, Quaid-i-Azam University, Islamabad, Pakistan
}

\begin{abstract}
The Bayesian analysis of the scale parameter of error function distribution has been considered in this paper. A class of informative and non-informative priors has been assumed to derive the corresponding posterior distributions. The Bayes estimators and associated risks have calculated under different loss functions. The Bayesian credible intervals have been constructed under each prior. The performance of the Bayes estimators have been evaluated and compared under a comprehensive simulation study. The purpose is to find out the combination of a loss function and a prior having the minimu m Bayes risk and hence producing the best results. The study depicts that in order to estimate the said parameter use of entropy loss function under informative priors can be preferred.
\end{abstract}

Keywords Squared Error Loss Function (SELF), Quadratic Loss Function (QLF), Entropy Loss Function (ELF), Precautionary Loss Function (PLF), Credible Intervals

\section{Introduction}

The error function distribution is one of the most widely used distributions in statistics. Estimating its parameter using Bayesian inference is extremely useful. Eberly and Casella[1] discussed the construction of Bayesian credible intervals using Rao-Blackwellized construction which offers smallest standard error of estimate. Kors gaard et al.[2] considered the multivariate normal distribution and concentrated on the model where residuals associated with liabilities of the binary traits have been assumed to be independent. A Bayesian analysis using Gibbs sampling has been outlined for the model where this assumption has been relaxed. Wang[3] proposed a criterion to choose a loss function in Bayesian analysis. Liang[4] introduced and derived Dempster EM-Algorithm for the two-component normal mixtu re models to obtain the iterative computation estimates, also used data augmentation and general Gibbs sampler to get the sample from posterior distribution under conjugate prior. Wang[5]developed the new method, called matrix-variate graphical models (MGGMs), which involves simultaneously modeling variable and sample dependencies with the matrix-normal distribution. Khan and Islam[6] evaluated the maintenance performance of the system when time is continuous and consider half-normal failure lifetime model as well as repair time model.

* Corresponding author:

navidferoz@hotmail.com (Navid Feroze)

Published online at http://journal.sapub.org/ijps

Copyright (C) 2012 Scientific \& Academic Publishing. All Rights Reserved
However, error function distribution has rarely received the attention of the analysts. But it is always of interest to study the behaviour and properties of the estimators for the parameters of the new/deprived distributions. So, the problem of estimation of the parameter of the error function distribution under a Bayesian framework has been addressed in this paper. A class of priors have been assumed under various loss functions to estimate the parameter of the distribution.

\section{Model and Likelihood Function}

The probability density function of error function distribution is:

$$
f(x)=\frac{\omega}{\sqrt{\pi}} e^{-\omega^{2} x^{2}} ;-\infty<x<\infty, \omega>0
$$

It is a special case of normal distribution with mean zero and considering $\frac{1}{\sqrt{2} \sigma}=\omega$ where $\sigma$ is the standard deviation of the normal distribution.

The like lihood function for a random sample of size ' $n$ ' is

$$
L(\omega \mid \underline{x}) \propto \omega^{n} e^{-\omega^{2} \sum_{i=1}^{n} x_{i}^{2}}
$$

\section{Bayesian Analysis under the Assumption of Uniform Prior}

The uniform prior is assumed to be: $p(\omega) \propto 1$ 
The posterior distribution under the assumption of uniform prior is:

$$
p(\omega \mid \underline{x})=\frac{2\left(\sum_{i=1}^{n} x_{i}^{2}\right)^{\frac{n+1}{2}}}{\Gamma\left(\frac{n+1}{2}\right)} \omega^{n} e^{-\omega^{2} \sum_{i=1}^{n} x_{i}^{2}} ; \omega>0
$$

The Bayes estimators and risks under SELF, QLF, ELF and PLF are respectively presented in the following.

$$
\begin{aligned}
& \omega_{S E L F}=\frac{\Gamma\left(\frac{n+2}{2}\right)}{\Gamma\left(\frac{n+1}{2}\right)\left(\sum_{i=1}^{n} x_{i}^{2}\right)^{\frac{1}{2}}} \\
& R\left(\omega_{\text {SELF }}\right)=\frac{n+1}{2\left(\sum_{i=1}^{n} x_{i}^{2}\right)}-\frac{1}{\left(\sum_{i=1}^{n} x_{i}^{2}\right)}\left\{\frac{\Gamma\left(\frac{n+2}{2}\right)}{\Gamma\left(\frac{n+1}{2}\right)}\right\}^{2} \\
& \omega_{Q L F}=\frac{(n-1) \Gamma\left(\frac{n}{2}\right)}{2 \Gamma\left(\frac{n+1}{2}\right)\left(\sum_{i=1}^{n} x_{i}^{2}\right)^{\frac{1}{2}}} \\
& R\left(\omega_{Q L F}\right)=1-\frac{(n-1)}{2}\left\{\frac{\Gamma\left(\frac{n}{2}\right)}{\Gamma\left(\frac{n+1}{2}\right)}\right\}^{2} \\
& \omega_{E L F}=\frac{\Gamma\left(\frac{n+1}{2}\right)}{\Gamma\left(\frac{n}{2}\right)\left(\sum_{i=1}^{n} x_{i}^{2}\right)^{\frac{1}{2}}} \\
& R\left(\omega_{E L F}\right)=\mathrm{E}(\ln \omega)-\ln \left(\omega_{E L F}\right) \\
& \omega_{P L F}=\left\{\frac{n+1}{2 \sum_{i=1}^{n} x_{i}^{2}}\right\}^{\frac{1}{2}} \\
& R\left(\omega_{P L F}\right)=2\left[\left\{\frac{n+1}{2 \sum_{i=1}^{n} x_{i}^{2}}\right\}^{\frac{1}{2}}-\frac{\Gamma\left(\frac{n+2}{2}\right)}{\Gamma\left(\frac{n+1}{2}\right)\left(\sum_{i=1}^{n} x_{i}^{2}\right)^{\frac{1}{2}}}\right]
\end{aligned}
$$

\section{Bayesian Analysis under the} Assumption of Jeffreys Prior

The Jeffreys prior is defined as:

$$
p_{j} \propto \sqrt{|I(\omega)|} \text { here; } p_{j} \propto \sqrt{|(\omega)|}=\frac{1}{\mu}
$$

The posterior distribution under Jeffreys prior is:

$$
p(\omega \mid \underline{x})=\frac{2\left(\sum_{i=1}^{n} x_{i}^{2}\right)^{\frac{n}{2}}}{\Gamma\left(\frac{n}{2}\right)} \omega^{n-1} e^{-\omega^{2} \sum_{i=1}^{n} x_{i}^{2}} ; \omega>0
$$

The Bayes estimators and risks under SELF, QLF, ELF and PLF are respectively given in the following.

$$
\begin{aligned}
& \omega_{S E L F}=\frac{\Gamma\left(\frac{n+1}{2}\right)}{\Gamma\left(\frac{n}{2}\right)\left(\sum_{i=1}^{n} x_{i}^{2}\right)^{\frac{1}{2}}} \\
& R\left(\omega_{\text {SELF }}\right)=\frac{n}{2\left(\sum_{i=1}^{n} x_{i}^{2}\right)}-\frac{1}{\left(\sum_{i=1}^{n} x_{i}^{2}\right)}\left\{\frac{\Gamma\left(\frac{n+1}{2}\right)}{\Gamma\left(\frac{n}{2}\right)}\right\}^{2} \\
& \omega_{Q L F}=\frac{(n-2) \Gamma\left(\frac{n-1}{2}\right)}{2 \Gamma\left(\frac{n}{2}\right)\left(\sum_{i=1}^{n} x_{i}^{2}\right)^{\frac{1}{2}}} \\
& R\left(\omega_{Q L F}\right)=1-\frac{(n-2)}{2}\left\{\frac{\Gamma\left(\frac{n-1}{2}\right)}{\Gamma\left(\frac{n}{2}\right)}\right\}^{2} \\
& \omega_{E L F}=\frac{\Gamma\left(\frac{n}{2}\right)}{\Gamma\left(\frac{n-1}{2}\right)\left(\sum_{i=1}^{n} x_{i}^{2}\right)^{\frac{1}{2}}} \\
& R\left(\omega_{E L F}\right)=\mathrm{E}(\ln \omega)-\ln \left(\omega_{E L F}\right) \\
& \omega_{P L F}=\left\{\frac{n}{2 \sum_{i=1}^{n} x_{i}^{2}}\right\}^{\frac{1}{2}}
\end{aligned}
$$




$$
R\left(\omega_{P L F}\right)=2\left[\left\{\frac{n}{2 \sum_{i=1}^{n} x_{i}^{2}}\right\}^{\frac{1}{2}}-\frac{\Gamma\left(\frac{n+1}{2}\right)}{\Gamma\left(\frac{n}{2}\right)\left(\sum_{i=1}^{n} x_{i}^{2}\right)^{\frac{1}{2}}}\right]
$$

\section{Bayesian Analysis under the Assumption of Maxwell Prior}

The Maxwell prior is assumed to be:

$$
p(\omega) \propto \omega^{2} e^{-\omega^{2} / a} \quad \omega>0, a>0
$$

Where ' $a$ ' is hyper-parameter

The posterior distribution under Maxwell prior is:

$$
p(\omega \mid \underline{x})=\frac{2\left(\sum_{i=1}^{n} x_{i}^{2}+\frac{1}{a}\right)^{\frac{n+3}{2}}}{\Gamma\left(\frac{n+3}{2}\right)} \omega^{n+2} e^{-\omega^{2}\left(\sum_{i=1}^{n} x_{i}^{2}+\frac{1}{a}\right)}
$$

The Bayes estimators and risks under SELF, QLF, ELF and PLF are respectively shown in the following.

$$
\begin{gathered}
\omega_{S E L F}=\frac{\Gamma\left(\frac{n+4}{2}\right)}{\Gamma\left(\frac{n+3}{2}\right)\left(\sum_{i=1}^{n} x_{i}^{2}+\frac{1}{a}\right)^{\frac{1}{2}}} \\
R\left(\omega_{S E L F}\right)=\frac{n+4}{\left.\sum_{i=1}^{n} x_{i}^{2}+\frac{1}{a}\right)}-\frac{1}{\left(\sum_{i=1}^{n} x_{i}^{2}+\frac{1}{a}\right)}\left\{\frac{\Gamma\left(\frac{n+4}{2}\right)}{\Gamma\left(\frac{n+3}{2}\right)}\right\}^{2} \\
R\left(\omega_{Q L F}\right)=1-\frac{(n+1) \Gamma\left(\frac{n+2}{2}\right)}{2}\left\{\frac{\left(\frac{n+3}{2}\right)\left(\sum_{i=1}^{n} x_{i}^{2}+\frac{1}{a}\right)^{\frac{1}{2}}}{\Gamma\left(\frac{n+3}{2}\right)}\right. \\
\omega_{E L F}=\frac{\Gamma\left(\frac{n+2}{2}\right)\left(\sum_{i=1}^{n} x_{i}^{2}+\frac{1}{a}\right)^{\frac{1}{2}}}{2} \\
\{
\end{gathered}
$$

$$
\begin{gathered}
R\left(\omega_{E L F}\right)=\mathrm{E}(\ln \omega)-\ln \left(\omega_{E L F}\right) \\
\omega_{P L F}=\left\{\frac{n+3}{2\left(\sum_{i=1}^{n} x_{i}^{2}+\frac{1}{a}\right)}\right\}^{\frac{1}{2}}
\end{gathered}
$$$$
R\left(\omega_{P L F}\right)=2\left[\left\{\frac{n+3}{2\left(\sum_{i=1}^{n} x_{i}^{2}+\frac{1}{a}\right)}\right\}^{\frac{1}{2}}-\frac{\Gamma\left(\frac{n+4}{2}\right)}{\Gamma\left(\frac{n+3}{2}\right)\left(\sum_{i=1}^{n} x_{i}^{2}+\frac{1}{a}\right)^{\frac{1}{2}}}\right]
$$

\section{Bayesian Analysis under the Assumption of Rayleigh Prior}

The Rayleigh prior is assumed to be:

$$
p(\omega) \propto \omega e^{\omega^{2} / b^{2}} \omega>0, b>0
$$

Where ' $b$ ' is hyper-parameter.

The posterior distribution under Ray leigh prior is:

$$
p(\omega \mid \underline{x})=\frac{2\left(\sum_{i=1}^{n} x_{i}^{2}+\frac{1}{b^{2}}\right)^{\frac{n+2}{2}}}{\Gamma\left(\frac{n+2}{2}\right)} \omega^{n+1} e^{-\omega^{2}\left(\sum_{i=1}^{n} x_{i}^{2}+\frac{1}{b^{2}}\right)} ;
$$

The Bayes estimators and risks under SELF, QLF, ELF and PLF are respectively presented in the following.

$$
\begin{gathered}
\omega_{S E L F}=\frac{\Gamma\left(\frac{n+3}{2}\right)}{\Gamma\left(\frac{n+2}{2}\right)\left(\sum_{i=1}^{n} x_{i}^{2}+\frac{1}{b^{2}}\right)^{\frac{1}{2}}} \\
R\left(\omega_{S E L F}\right)=\frac{n+3}{2\left(\sum_{i=1}^{n} x_{i}^{2}+\frac{1}{b^{2}}\right)}-\frac{1}{\left(\sum_{i=1}^{n} x_{i}^{2}+\frac{1}{b^{2}}\right)}\left\{\frac{\Gamma\left(\frac{n+3}{2}\right)}{\Gamma\left(\frac{n+2}{2}\right)}\right\}^{2} \\
\omega_{Q L F}=\frac{\left(\frac{n+1}{2}\right)}{2 \Gamma\left(\frac{n+2}{2}\right)\left(\sum_{i=1}^{n} x_{i}^{2}+\frac{1}{b^{2}}\right)^{\frac{1}{2}}}
\end{gathered}
$$




$$
\begin{gathered}
R\left(\omega_{Q L F}\right)=1-\frac{(n)}{2}\left\{\frac{\Gamma\left(\frac{n+1}{2}\right)}{\Gamma\left(\frac{n+2}{2}\right)}\right\}^{2} \\
\omega_{E L F}=\frac{\Gamma\left(\frac{n+2}{2}\right)}{R\left(\omega_{E L F}\right)=\mathrm{E}(\ln \omega)-\ln \left(\omega_{E L F}\right)} \\
\omega_{P L F}=\left\{\frac{n+1}{\left.\sum_{i=1}^{2} x_{i}^{2}+\frac{1}{b^{2}}\right)^{\frac{1}{2}}}\right. \\
\left.\left.\left.R\left(\omega_{P L F}\right)=2\left[\left\{\frac{n+2}{2\left(\sum_{i=1}^{n} x_{i}^{2}+\frac{1}{b^{2}}\right)}\right\}^{\frac{1}{2}}\right\}^{\frac{1}{2}} x_{i}^{2}+\frac{1}{b^{2}}\right)\right\}^{\Gamma\left(\frac{n+2}{2}\right)\left(\sum_{i=1}^{n} x_{i}^{2}+\frac{1}{b^{2}}\right)^{\frac{1}{2}}}\right]
\end{gathered}
$$

\section{Bayesian Analysis under the Assumption of Chi Prior}

The chi prior is assumed to be:

$$
p(\omega) \propto \omega^{k-1} e^{\omega^{2} / 2} \omega>0, \quad k>0
$$

Where ' $k$ ' is hyper-parameter.

The posterior distribution under chi prior is:

$$
p(\omega \mid \underline{x})=\frac{2\left(\sum_{i=1}^{n} x_{i}^{2}+\frac{1}{2}\right)^{\frac{n+k}{2}}}{\Gamma\left(\frac{n+k}{2}\right)} \omega^{n+k} e^{-\omega^{2}\left(\sum_{i=1}^{n} x_{i}^{2}+\frac{1}{2}\right)}
$$

The Bayes estimators and risks under SELF, QLF, ELF and PLF are respectively derived in the following.

$$
\begin{gathered}
\omega_{\text {SELF }}=\frac{\Gamma\left(\frac{n+k+1}{2}\right)}{\Gamma\left(\frac{n+k}{2}\right)\left(\sum_{i=1}^{n} x_{i}^{2}+\frac{1}{2}\right)^{\frac{1}{2}}} \\
R\left(\omega_{\text {SELF }}\right)=\frac{n+k}{2\left(\sum_{i=1}^{n} x_{i}^{2}+\frac{1}{2}\right)}-\frac{1}{\left(\sum_{i=1}^{n} x_{i}^{2}+\frac{1}{2}\right)}\left\{\frac{\Gamma\left(\frac{n+k+2}{2}\right)}{\Gamma\left(\frac{n+k+1}{2}\right)}\right\}^{2}
\end{gathered}
$$

$$
\begin{aligned}
& \omega_{Q L F}=\frac{(n+k-2) \Gamma\left(\frac{n+k-1}{2}\right)}{2 \Gamma\left(\frac{n+k}{2}\right)\left(\sum_{i=1}^{n} x_{i}^{2}+\frac{1}{2}\right)^{\frac{1}{2}}} \\
& R\left(\omega_{Q L F}\right)=1-\frac{(n+k-2)}{2}\left\{\frac{\Gamma\left(\frac{n+k-1}{2}\right)}{\Gamma\left(\frac{n+k}{2}\right)}\right\}^{2} \\
& \omega_{E L F}=\frac{\Gamma\left(\frac{n+k}{2}\right)}{\Gamma\left(\frac{n+k-1}{2}\right)\left(\sum_{i=1}^{n} x_{i}^{2}+\frac{1}{2}\right)^{\frac{1}{2}}} \\
& R\left(\omega_{E L F}\right)=\mathrm{E}(\ln \omega)-\ln \left(\omega_{E L F}\right) \\
& \omega_{P L F}=\left\{\frac{n+k}{2\left(\sum_{i=1}^{n} x_{i}^{2}+\frac{1}{2}\right)}\right\}^{\frac{1}{2}} \\
& R\left(\omega_{P L F}\right)=2\left[\left\{\frac{n+k}{2\left(\sum_{i=1}^{n} x_{i}^{2}+\frac{1}{2}\right)}\right\}^{\frac{1}{2}}-\frac{\Gamma\left(\frac{n+k+1}{2}\right)}{\Gamma\left(\frac{n+k}{2}\right)\left(\sum_{i=1}^{n} x_{i}^{2}+\frac{1}{2}\right)^{\frac{1}{2}}}\right]
\end{aligned}
$$

8. Bayesian Analysis under the Assumption of Normal Prior Considering Location Parameter to be Zero

The normal prior is assumed to be:

$$
p(\omega) \propto \omega^{k-1} e^{\omega^{2} / 2 h^{2}} \quad \omega>0 ; \quad h>0
$$

Where ' $h$ ' is hyper-parameter.

The posterior distribution under normal prior is:

$$
p(\omega \mid \underline{x})=\frac{2\left(\sum_{i=1}^{n} x_{i}^{2}+\frac{1}{2 h^{2}}\right)^{\frac{n+1}{2}}}{\Gamma\left(\frac{n+1}{2}\right)} \omega^{n} e^{-\omega^{2}\left(\sum_{i=1}^{n} x_{i}^{2}+\frac{1}{2 h^{2}}\right)} ;
$$

The Bayes estimators and risks under SELF, QLF, ELF and PLF are respectively presented in the following. 


$$
\begin{aligned}
& \omega_{S E L F}=\frac{\Gamma\left(\frac{n+2}{2}\right)}{1} \\
& \Gamma\left(\frac{n+1}{2}\right)\left(\sum_{i=1}^{n} x_{i}^{2}+\frac{1}{2 h^{2}}\right)^{\frac{1}{2}} \\
& R\left(\omega_{\text {SELF }}\right)=\frac{n+1}{2\left(\sum_{i=1}^{n} x_{i}^{2}+\frac{1}{2 h^{2}}\right)}-\frac{1}{\left(\sum_{i=1}^{n} x_{i}^{2}+\frac{1}{2 h^{2}}\right)}\left\{\frac{\Gamma\left(\frac{n+2}{2}\right)}{\Gamma\left(\frac{n+1}{2}\right)}\right\}^{2} \\
& (n-1) \Gamma\left(\frac{n}{2}\right) \\
& \omega_{Q L F}=\frac{2 \Gamma\left(\frac{n+1}{2}\right)\left(\sum_{i=1}^{n} x_{i}^{2}+\frac{1}{2 h^{2}}\right)^{\frac{1}{2}}}{2} \\
& R\left(\omega_{Q L F}\right)=1-\frac{(n-1)}{2}\left\{\frac{\Gamma\left(\frac{n}{2}\right)}{\Gamma\left(\frac{n+1}{2}\right)}\right\}^{2} \\
& \omega_{E L F}=\frac{\Gamma\left(\frac{n+1}{2}\right)}{\Gamma\left(\frac{n}{2}\right)\left(\sum_{i=1}^{n} x_{i}^{2}+\frac{1}{2 h^{2}}\right)^{\frac{1}{2}}} \\
& R\left(\omega_{E L F}\right)=\mathrm{E}(\ln \omega)-\ln \left(\omega_{E L F}\right) \\
& \omega_{P L F}=\left\{\frac{n+1}{2\left(\sum_{i=1}^{n} x_{i}^{2}+\frac{1}{2 h^{2}}\right)}\right\}^{\frac{1}{2}}
\end{aligned}
$$

\section{Bayesian Credible Intervals under Different Priors}

The Bayesian credible intervals, as discussed by Saleem and Raza[7], under uniform, Jeffreys, Maxwell, Rayleigh, chi and normal priors are respectively constructed in the following.

$$
\frac{2\left(\sum_{i=1}^{n} x_{i}^{2}\right)}{\chi_{2\left(\frac{n+1}{2}\right),\left(\frac{\alpha}{2}\right)}^{2}}<\omega_{U}<\frac{2\left(\sum_{i=1}^{n} x_{i}^{2}\right)}{\chi_{2\left(\frac{n+1}{2}\right),\left(1-\frac{\alpha}{2}\right)}^{2}}
$$

$$
\begin{aligned}
& \frac{2\left(\sum_{i=1}^{n} x_{i}^{2}\right)}{\chi_{2\left(\frac{n}{2}\right),\left(\frac{\alpha}{2}\right)}^{2}}<\omega_{J}<\frac{2\left(\sum_{i=1}^{n} x_{i}^{2}\right)}{\chi_{2\left(\frac{n}{2}\right),\left(1-\frac{\alpha}{2}\right)}^{2}} \\
& \frac{2\left(\sum_{i=1}^{n} x_{i}^{2}+\frac{1}{a}\right)}{\chi_{2\left(\frac{n+3}{2}\right),\left(\frac{\alpha}{2}\right)}^{2}}<\omega_{M}<\frac{2\left(\sum_{i=1}^{n} x_{i}^{2}+\frac{1}{a}\right)}{\chi_{2\left(\frac{n+3}{2}\right),\left(1-\frac{\alpha}{2}\right)}^{2}} \\
& \frac{2\left(\sum_{i=1}^{n} x_{i}^{2}+\frac{1}{b^{2}}\right)}{\chi_{2\left(\frac{n+2}{2}\right),\left(\frac{\alpha}{2}\right)}^{2}}<\omega_{R}<\frac{2\left(\sum_{i=1}^{n} x_{i}^{2}+\frac{1}{b^{2}}\right)}{\chi_{2\left(\frac{n+2}{2}\right),\left(1-\frac{\alpha}{2}\right)}^{2}} \\
& \frac{2\left(\sum_{i=1}^{n} x_{i}^{2}+\frac{1}{2}\right)}{\chi_{2\left(\frac{n+k}{2}\right),\left(\frac{\alpha}{2}\right)}^{2}}<\omega_{C}<\frac{2\left(\sum_{i=1}^{n} x_{i}^{2}+\frac{1}{2}\right)}{\chi_{2\left(\frac{n+k}{2}\right),\left(1-\frac{\alpha}{2}\right)}^{2}} \\
& \frac{2\left(\sum_{i=1}^{n} x_{i}^{2}+\frac{1}{2 h^{2}}\right)}{\chi_{2\left(\frac{n+1}{2}\right),\left(\frac{\alpha}{2}\right)}^{2}}<\omega_{N}<\frac{2\left(\sum_{i=1}^{n} x_{i}^{2}+\frac{1}{2 h^{2}}\right)}{\chi_{2\left(\frac{n+1}{2}\right),\left(1-\frac{\alpha}{2}\right)}^{2}}
\end{aligned}
$$

\section{Simulation Study}

Simu lation study has been carried out using $\mathrm{n}=50,100,200$ and 300 for $\omega \in(1,2,3,4,5,6)$; however, the results are presented for $\omega=1$. While, the discussions have been made for all $\omega \in(1,2,3,4,5,6)$. Different values of the hyper-parameters have been used and the results for the values giving better convergence and the minimum risks have been presented. In order to have more precise estimates, the results have been replicated sufficiently. The risks associated with Bayes estimates have been underlined in the tables. Similarly, the differences between lower and upper limits of credible intervals have been underlined.

Table 1. Bayes est imates and risks under uniform prior

\begin{tabular}{|c|c|c|c|c|}
\hline \multirow{2}{*}{$\mathrm{n}$} & \multicolumn{4}{|c|}{ Loss Functions } \\
\cline { 2 - 5 } & SELF & QLF & ELF & PLF \\
\hline \multirow{2}{*}{50} & 1.38112 & 1.35350 & 1.36738 & 1.38790 \\
& 0.01879 & 0.01015 & 0.00503 & 0.01357 \\
\hline \multirow{2}{*}{100} & 1.15111 & 1.13960 & 1.14537 & 1.15396 \\
& 0.00657 & 0.00504 & 0.00250 & 0.00570 \\
\hline \multirow{2}{*}{200} & 1.00946 & 1.00441 & 1.00693 & 1.01071 \\
& 0.00254 & 0.00251 & 0.00124 & 0.00251 \\
\hline \multirow{2}{*}{300} & 1.00377 & 1.00043 & 1.00210 & 1.00461 \\
& 0.00167 & 0.00167 & 0.00083 & 0.00167 \\
\hline
\end{tabular}


Table 2. Bayes est imates and risks under Jeffreys prior

\begin{tabular}{|c|c|c|c|c|}
\hline \multirow{2}{*}{$\mathrm{n}$} & \multicolumn{4}{|c|}{ Loss Functions } \\
\cline { 2 - 5 } & SELF & QLF & ELF & PLF \\
\hline \multirow{2}{*}{50} & 1.36738 & 1.33948 & 1.35349 & 1.37423 \\
& 0.01879 & 0.01036 & 0.00513 & 0.01371 \\
\hline \multirow{2}{*}{100} & 1.14537 & 1.13380 & 1.13960 & 1.14824 \\
& 0.00658 & 0.00509 & 0.00252 & 0.00574 \\
\hline \multirow{2}{*}{200} & 1.00693 & 1.00187 & 1.00441 & 1.00819 \\
& 0.00254 & 0.00252 & 0.00125 & 0.00252 \\
\hline \multirow{2}{*}{300} & 1.00210 & 0.99875 & 1.00043 & 1.00294 \\
& 0.00168 & 0.00168 & 0.00083 & 0.00167 \\
\hline
\end{tabular}

Table 3. Bayes estimates and risks under Maxwell prior

\begin{tabular}{|c|c|c|c|c|}
\hline \multirow{2}{*}{$\mathrm{n}$} & \multicolumn{4}{|c|}{ Loss Functions } \\
\cline { 2 - 5 } & SELF & QLF & ELF & PLF \\
\hline \multirow{2}{*}{50} & 1.31254 & 1.28730 & 1.29998 & 1.31874 \\
& 0.04914 & 0.00975 & 0.00484 & 0.01241 \\
\hline \multirow{2}{*}{100} & 1.13302 & 1.12191 & 1.12747 & 1.13577 \\
& 0.01877 & 0.00493 & 0.00245 & 0.00550 \\
\hline \multirow{2}{*}{200} & 1.00432 & 0.99935 & 1.00184 & 1.00556 \\
& 0.00747 & 0.00248 & 0.00123 & 0.00248 \\
\hline \multirow{2}{*}{300} & 1.00042 & 0.99711 & 0.99877 & 1.00125 \\
& 0.00496 & 0.00166 & 0.00082 & 0.00165 \\
\hline
\end{tabular}

Table 4. Bayes est imates and risks under Rayleigh prior

\begin{tabular}{|c|c|c|c|c|}
\hline \multirow{2}{*}{$\mathrm{n}$} & \multicolumn{4}{|c|}{ Loss Functions } \\
\cline { 2 - 5 } & SELF & QLF & ELF & PLF \\
\hline \multirow{2}{*}{50} & 1.22224 & 1.19827 & 1.21032 & 1.22813 \\
& 0.04344 & 0.00995 & 0.00493 & 0.01178 \\
\hline \multirow{2}{*}{100} & 1.10025 & 1.08936 & 1.09482 & 1.10295 \\
& 0.01788 & 0.00499 & 0.00247 & 0.00540 \\
\hline \multirow{2}{*}{200} & 0.99200 & 0.98707 & 0.98954 & 0.99323 \\
& 0.00732 & 0.00250 & 0.00124 & 0.00246 \\
\hline \multirow{2}{*}{300} & 0.99222 & 0.98893 & 0.99058 & 0.99304 \\
& 0.00490 & 0.00167 & 0.00083 & 0.00164 \\
\hline
\end{tabular}

Table 5. Bayes est imates and risks under chi prior

\begin{tabular}{|c|c|c|c|c|}
\hline \multirow{2}{*}{$\mathrm{n}$} & \multicolumn{4}{|c|}{ Loss Functions } \\
\cline { 2 - 5 } & SELF & QLF & ELF & PLF \\
\hline \multirow{2}{*}{50} & 1.36911 & 1.34226 & 1.35575 & 1.37571 \\
& 0.01829 & 0.00995 & 0.00493 & 0.01320 \\
\hline \multirow{2}{*}{100} & 1.14927 & 1.13789 & 1.14360 & 1.15209 \\
& 0.00653 & 0.00499 & 0.00247 & 0.00564 \\
\hline \multirow{2}{*}{200} & 1.00941 & 1.00439 & 1.00690 & 1.01066 \\
& 0.00253 & 0.00250 & 0.00124 & 0.00250 \\
\hline \multirow{2}{*}{300} & 1.00376 & 1.00043 & 1.00210 & 1.00459 \\
& 0.00167 & 0.00167 & 0.00083 & 0.00166 \\
\hline
\end{tabular}

From the above study it can be seen that by increasing the sample size the estimated value of the parameter converges to the true value of the parameter and magnitude of risk associated with each estimate decreases. The increasing values of the parameter impose a negative impact on rate of convergence under each prior; similarly, the performance of squared error loss function and precautionary loss function is badly affected. However, the performance of quadratic loss function and entropy loss function is independent of choice of parametric value. In co mparis on of non-informative priors the uniform prior gives the better estimates as the corresponding risks are smaller for each loss function. While in case of informative priors the Maxwell prior for QLF and ELF, Chi prior for SELF and Rayleigh prior for PLF provide the best results. Similarly, estimates under entropy loss function give the minimum risks among all loss functions for each prior. It can also be assessed that the performance of estimates under informative priors is better than those under non-informative priors. So me prior elicitation technique may further strengthen this argument. Hence, the use of Maxwell prior under entropy loss function can be preferred to estimate the parameter of the error function distribution using a Bayesian framework.

In case of interval estimation, the credible intervals under uniform prior are again narrower than those under Jeffreys prior. Using informative priors, the intervals under chi prior are having the minimum width. So for Bayesian interval estimation of the parameter of error function distribution, the use of chi prior can be preferred.

Table 6. Bayes est imat es and risks under normal prior

\begin{tabular}{|c|c|c|c|c|}
\hline \multirow{2}{*}{$\mathrm{n}$} & \multicolumn{4}{|c|}{ Loss Functions } \\
\cline { 2 - 5 } & SELF & QLF & ELF & PLF \\
\hline \multirow{2}{*}{50} & 1.28730 & 1.26155 & 1.27449 & 1.29362 \\
& 0.01632 & 0.01015 & 0.00503 & 0.01265 \\
\hline \multirow{2}{*}{100} & 1.12191 & 1.11069 & 1.11631 & 1.12469 \\
& 0.00624 & 0.00504 & 0.00250 & 0.00556 \\
\hline \multirow{2}{*}{200} & 0.99935 & 0.99435 & 0.99685 & 1.00059 \\
& 0.00249 & 0.00251 & 0.00124 & 0.00249 \\
\hline \multirow{2}{*}{300} & 0.99711 & 0.99379 & 0.99545 & 0.99794 \\
& 0.00165 & 0.00167 & 0.00083 & 0.00166 \\
\hline
\end{tabular}

Table 7. $95 \%$ credible intervals under uniform and Jeffreys priors

\begin{tabular}{|c|c|c|c|c|}
\hline \multirow{2}{*}{$\mathrm{n}$} & \multicolumn{2}{|c|}{ Uniform Prior } & \multicolumn{2}{c|}{ Jeffreys Prior } \\
\cline { 2 - 5 } & lower Limit & Upper Limit & lower Limit & Upper Limit \\
\hline \multirow{2}{*}{50} & 0.57460 & 1.01838 & 0.56071 & 1.00824 \\
\cline { 2 - 5 } & \multicolumn{2}{|c|}{0.44378} & \multicolumn{2}{|c|}{0.44754} \\
\hline \multirow{2}{*}{100} & 0.58031 & 1.01017 & 0.58541 & 1.02189 \\
\cline { 2 - 5 } & \multicolumn{2}{|c|}{0.42986} & \multicolumn{2}{|c|}{0.43648} \\
\hline \multirow{2}{*}{200} & 0.81254 & 1.20248 & 0.81624 & 1.20915 \\
\cline { 2 - 5 } & \multicolumn{2}{|c|}{0.38994} & \multicolumn{2}{c|}{0.39290} \\
\hline \multirow{2}{*}{300} & 0.85001 & 1.16999 & 0.85244 & 1.17460 \\
\cline { 2 - 5 } & \multicolumn{2}{|c|}{0.31999} & \multicolumn{2}{c|}{0.32216} \\
\hline
\end{tabular}

Table 8. $95 \%$ credible intervals under Maxwell and Rayleigh priors

\begin{tabular}{|c|c|c|c|c|}
\hline \multirow{2}{*}{$\mathrm{n}$} & \multicolumn{2}{|c|}{ Maxwell Prior } & \multicolumn{2}{c|}{ Rayleigh Prior } \\
\cline { 2 - 5 } & lower Limit & Upper Limit & lower Limit & Upper Limit \\
\hline \multirow{2}{*}{50} & 0.55633 & 1.02635 & 0.46709 & 1.01495 \\
\cline { 2 - 5 } & \multicolumn{2}{|c|}{0.47001} & \multicolumn{2}{c|}{0.54786} \\
\hline \multirow{2}{*}{100} & 0.60046 & 1.03955 & 0.63598 & 1.10403 \\
\cline { 2 - 5 } & \multicolumn{2}{|c|}{0.43908} & \multicolumn{2}{c|}{0.46805} \\
\hline \multirow{2}{*}{200} & 0.82161 & 1.21354 & 0.84176 & 1.24451 \\
\cline { 2 - 5 } & \multicolumn{2}{|c|}{0.39193} & \multicolumn{2}{c|}{0.40275} \\
\hline \multirow{2}{*}{300} & 0.85652 & 1.17645 & 0.87033 & 1.19668 \\
\cline { 2 - 5 } & \multicolumn{2}{|c|}{0.31993} & \multicolumn{2}{c|}{0.32636} \\
\hline
\end{tabular}


Table 9. $95 \%$ credible intervals under chi and normal priors

\begin{tabular}{|c|c|c|c|c|}
\hline \multirow{2}{*}{$\mathrm{n}$} & \multicolumn{2}{|c|}{ Chi Prior } & \multicolumn{2}{|c|}{ NormalPrior } \\
\hline & lower Limit & Upper Limit & lower Limit & Upper Limit \\
\hline \multirow{2}{*}{50} & 0.57225 & 1.00888 & 0.51969 & 1.01900 \\
\hline & \multicolumn{2}{|c|}{0.43662} & \multicolumn{2}{|c|}{0.49931} \\
\hline \multirow{2}{*}{100} & 0.58289 & 1.01186 & 0.61092 & 1.06344 \\
\hline & \multicolumn{2}{|c|}{0.42897} & \multicolumn{2}{|c|}{0.45253} \\
\hline \multirow{2}{*}{200} & 0.81299 & 1.20197 & 0.82906 & 1.22693 \\
\hline & \multicolumn{2}{|c|}{0.38898} & \multicolumn{2}{|c|}{0.39787} \\
\hline \multirow{2}{*}{300} & 0.85043 & 1.16933 & 0.86141 & 1.18569 \\
\hline & \multicolumn{2}{|c|}{0.31890} & \multicolumn{2}{|c|}{0.32428} \\
\hline
\end{tabular}

\section{Conclusions and Recommendations}

The study has been conducted to estimate the parameter of the error function distribution using four different loss functions and under six informative and non-informative priors. The study indicates that for Bayesian point estimation, the use of entropy loss function under Maxwell prior can be preferred. While for interval estimation, the chi prior can affectively be emp loyed.

The study can be extended by using more priors and loss functions. Some censoring procedures and finite mixture of components of error function distribution can also be used.

\section{REFERENCES}

[1] L. E Eberly, G. Casella, "Estimating Bayesian credible intervals", Journal of Statistical Planning and Inference, vol.112, pp.115-132, 2003.

[2] R. I. Korsgaard, S. M. Lunda, D. Sorensen, et al., "Multivariate Bayesian analy sis of Gaussian, right censored Gaussian, ordered categorical and binary traits using Gibbs sampling", Genet. Sel. Evol., vol.35, pp. 159-183, 2003.

[3] H. Wang, "Bayesian analysis of Gaussian graphical models for correlated sample", Preprint submitted to Elsevier, 2011.

[4] L. Liang, "On simulation methods for two component normal mixture models under Bayesian approach", Uppsala Universitet, Project Report, 2009.

[5] L. Wang, "A note on the choice between two loss functions in Bayesian analysis", Soochow Journal of Mathematics, vol.31, no.3, pp. 30-307, 2005.

[6] M. A. Khan, H. M. Islam, "Bayesian analysis of system availability with half-normal life time", Quality Technology \& Quantitative Management, vol.9, no.2, pp. 203-209, 2012.

[7] M. Saleem, A. Raza, "On Bayesian analysis of the exponential survival time assuming the exponential censor time", Pakistan Journal of Science, vol.63, no.1, pp. 44-48, 2011.. 